\title{
APRENDIENDO EL VALOR DEL TIEMPO EN EDUCACIÓN FÍSICA
}

APRENDENDO O VALOR DO TEMPO NA EDUCAÇÃO FÍSICA Cृ

LEARNING THE VALUE OF TIME IN PHYSICAL EDUCATION C己

doi' https://doi.org/10.22456/1982-8918.112146

Javier Gil Quintana* <javier.gil-quintana@uv.es>

Joseba Etxebeste Otegi** <joseba.etxebeste@ehu.eus>

*Universitat de Valencia. Valencia, España.

**Universidad del País Vasco (UPV-EHU). Vitoria-Gasteiz, España.

Resumen: El presente artículo analiza las prácticas pedagógicas de la Educación Física de un contexto específico para describir cómo promueven el aprendizaje del tiempo. A partir de una etnografía se obtienen las actividades propuestas por el profesorado de Educación Física de una escuela de Meliana, en Valencia (España). Se recogen 82 tareas motrices que son analizadas y contextualizadas en el marco educativo. Los resultados, a la luz de las ciencias de la educación, muestran una tendencia a realizar tareas sin memoria de resultado en las que no existe un final marcado por las reglas y, en menor tiempo, actividades con memoria en las que los participantes se clasifican en función del desenlace. La escuela guía al alumnado a aprender a gestionar libremente el tiempo y las relaciones socioafectivas que tienen lugar en él, a adaptarse a los ritmos sociales impuestos y las emociones derivadas de estos.

Palabras clave: Educación Física. Tiempo. Ejercicio físico. Emociones expresadas.
Recibido en: 18 nov. 2020 Aprobado en: 14 jul. 2021 Publicado en: 18 sep. 2021

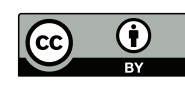

Este es un artículo publicado bajo la licencia Creative Commons Atribución 4.0 Internacional (CC BY 4.0).

eISSN: $1982-8918$ 


\section{INTRODUCCIÓN}

El alumnado aprende a ir a la escuela el mes que comienza el curso escolar, a entrar a clase a las nueve de la mañana y a salir a las cuatro de la tarde, a permanecer en el aula durante horas, a gozar de 30 minutos de recreo, a estudiar para los exámenes en diciembre, marzo y junio, a disfrutar de vacaciones en Navidad, Semana Santa y verano. En resumen: a repetir las secuencias temporales propias de la escolarización obligatoria.

Día tras día en la escuela, los niños y niñas aprenden el valor del tiempo (DOMÈNECH, 2011), a darle sentido a las cadencias, duraciones y velocidades. Gracias a sus vivencias asimilan cómo actuar, cómo organizarse, qué acciones realizar, cómo relacionarse o qué espacios ocupar en función del tiempo en patios y aulas (GIMENO, 2008). La escuela moldea el proceso de socialización, la socioafectividad, la manera de relacionarse con el entorno físico, social y cultural y todo ello bajo el movimiento de las agujas del reloj.

El tiempo es, para la Física, una magnitud vinculada a la velocidad, al espaciotiempo. Desde un punto de vista cultural, el tiempo es una construcción social (DOMĖNECH, 2011; GIMENO, 2008). Está tan anclado en la subjetividad que se olvida que se necesita toda una vida en aprenderlo. Como dice Norbert Elias (1989, p. 20) cada individuo "[...] aprende desde su infancia tanto el concepto de tiempo como la institución social del tiempo que le está unida de modo indisoluble". El sociólogo recuerda que, de manera consciente o inconsciente, cada persona aprende cómo es el tiempo de producción, el del ocio, el de la vida privada, el de las relaciones sociales, el del cuidado de menores o el de las tareas domésticas. Cada forma de tiempo es reflejo de la sociedad, lo que permite a cada sujeto "[...] ir creciendo en una sociedad a la cual pertenecen ese concepto y esa institución” (ELIAS, 1989, p. 20).

El aprendizaje del tiempo ocurre de manera oculta, sin que se tenga conciencia de sus efectos. El tiempo es intangible, es una variable como señala Elias (1989, p.10) que no se puede "[...] ver ni sentir, ni escuchar ni gustar ni olfatear". A diferencia de otros aspectos que se pueden ver, tocar, coger o manipular, el tiempo es invisible, intocable, transparente. Puede pasar desapercibido, pero siempre está ahí: lo que pone en evidencia la dificultad de reflexionar sobre él y sus efectos. Como dice Gimeno (2008, p. 27) el tiempo supone "[...] un factor socializador casi invisible de los individuos, pero muy efectivo". Una vez que el tiempo es aprendido por los miembros de una comunidad "[...] se convierte en algo tan íntimo y evidente que se olvida que está construido socialmente (ETXEBESTE et al., 2014, p. 36).

Parece razonable estudiar las características del tiempo en la escuela y en sus consecuencias sobre los discentes, sacar a la luz el currículum oculto del tiempo en el proceso de socialización. Por ello el presente artículo analiza las prácticas pedagógicas de la Educación Física para describir cómo promueven el aprendizaje del tiempo. Se hace necesario saber qué estructuras temporales asimila el alumnado, qué afectividad y sociabilidad desarrolla en función del tiempo y qué consecuencias pedagógicas tiene. Hacerlo podría dilucidar cómo la escuela cumple con los fines pedagógicos que le han sido encomendados. Es necesario destapar, como dice 
Gimeno (2008), esa caja negra llamada tiempo, hacer una reflexión a fondo sobre su utilización y las posibilidades que lo delimitan (DOMĖNECH, 2011). El tiempo influye en la formación de la ciudadanía y es un compromiso que la educación no puede dejar de asumir.

El tiempo cultural puede ser definido como "la capacidad de los seres humanos de identificar en el flujo de los acontecimientos dos o más eventos relacionándolos entre sí y utilizando uno de ellos como referencia" (ELIAS, 1989, p.207). El estudio comportamental de los fenómenos naturales y su clasificación (ROSENBLUETH; WIENER; BIGELOW, 1943) permite diferenciar dos grandes grupos de relación entre acontecimientos: por un lado, los causales o con propósito; y por otro, los no causales o carentes de propósito. Para los autores, dos eventos tienen propósito o son causales cuando uno de ellos produce el siguiente; y carecen de él cuando no existe causalidad entre ambos, esto es, cuando un evento no produce el siguiente. Un reloj-alarma tiene intencionalidad, genera un nuevo comportamiento, una nueva acción, el sonar del despertador; mientras que el reloj sin alarma carece de ella, no tiene intención ni propósito, ya que no genera nada nuevo (ELIAS, 1989).

\section{EDUCACIÓN FÍSICA Y TIEMPO}

Como parte del universo educativo, la Educación Física también contribuye en la interiorización del valor del tiempo. La asignatura orienta al alumnado a que aprenda a organizarse, actuar, relacionarse y emocionarse en función del contexto social, pero también temporal, en el que se encuentra.

En España, la Educación física es una asignatura obligatoria en el currículum escolar que ocupa dos o tres horas del horario escolar en función de la Comunidad Autónoma y de la etapa escolar. Está regida por la Ley Orgánica 2/2006, de 3 de mayo, de Educación, consolidada a su vez por la Ley Orgánica 3/2020.

La Educación Física ha tratado el tiempo desde la visión de la Física, desde la percepción de la velocidad de desplazamiento de los objetos, vinculando los conceptos de espacio-tiempo (ETXEBESTE et al., 2014). La asignatura ha priorizado aspectos ligados al cálculo de la continuidad, la duración, la secuencia o el ritmo para optimizar capacidades como la coordinación de los segmentos corporales o la precisión para trazar una trayectoria con un objeto en el aire, propias de métodos tradicionales como la Psicomotricidad o la Psicocinética de LeBoulch (1987). Asimismo, la asignatura ha hecho hincapié en muchas ocasiones en el desarrollo de capacidades asociadas a un tiempo físico como la velocidad de reacción en décimas de segundo, la rapidez para recorrer dos puntos separados o el número de pulsaciones cardíacas por minuto, propias del común método deportivo.

Si bien los aprendizajes asociados al espacio-tiempo en la Educación Física son de una utilidad evidente, su aplicación se restringe a la percepción de la realidad física. La asignatura ha de tener también en cuenta la percepción social del tiempo, la experiencia vivida de los sujetos ya que influye en las conductas motrices del alumnado y ello supone modelar su dimensión biológica, física y cognitiva sin olvidar la social y la afectiva (PARLEBAS, 2001). La Educación Física debe impulsar el 
desarrollo de capacidades asociadas a un tiempo físico sin olvidar la interpretación sociocultural y la vivencia individual. Es necesario que la asignatura sumerja el tiempo físico en el contexto social para conocer su efecto socializador y para dar cuenta de la realidad oculta de la cultura del tiempo (ETXEBESTE et al., 2014).

Las tareas motrices de la Educación Física, como acciones profundamente enraizadas con la cultura en la que se desarrollan, permiten guiar al alumnado a interiorizar el valor social del tiempo. Estas poseen una temporalidad definida culturalmente, se insertan "en una temporalidad construida por los jugadores y por las instituciones" (PARLEBAS, 2001, p. 445) y permiten incorporar las distintas percepciones temporales de ese contexto. Los participantes son orientados a interiorizar las temporalidades socialmente construidas cuando participan en distintas disciplinas deportivas (LEVEL, 2011). Así lo muestra por ejemplo Wacquant (2002) en su estudio sobre actividades en el gimnasio, describiendo cómo están regidas por un tempo social que es incorporado por los deportistas a partir de su participación.

Sabiendo que las tareas motrices son espejo de la cultura e impulsan el aprendizaje social del tiempo en la escuela, este trabajo tiene como objetivo analizar las prácticas pedagógicas de la Educación Física de un contexto específico para describir cómo promueven el aprendizaje del tiempo. Es una meta que comienza a dar sus primeros pasos en educación (DOMĖNECH, 2011; GIMENO, 2008) y que sin duda también sería recomendable extender a la Educación Física para desvelar su oscuro, pero implacable, currículo oculto (ETXEBESTE et al., 2014; GIL, 2017).

\section{TIEMPO, TAREAS MOTRICES Y AFECTIVIDAD}

Saber cómo las tareas motrices impulsan la interiorización socioafectiva del tiempo exige conocer las características temporales de las tareas motrices que se realizan en las clases de Educación Física, analizar si poseen propósito o carecen de él (ETXEBESTE, 2013). Cada tarea posee una lógica interna, un sistema de obligaciones y derechos impuesto por las normas del juego deportivo, de la tarea motriz, y que orienta la adquisición de modelos de comportamientos entre las personas, en el espacio, la cultura material y, como no, el tiempo ludo-deportivo (PARLEBAS, 2001).

Primeramente, habrá que identificar dos eventos lúdicos y estudiar la relación que se establece entre ellos, la presencia o ausencia de causalidad o propósito. Suits (1978) avanza dos conceptos como son el de juego abierto y juego cerrado en cuanto a la presencia o ausencia de propósito de los eventos. Para el autor "serán juegos abiertos los que no tengan un propósito inherente que al conseguirse suponga el fin del juego [...]; y serán juegos cerrados cuando posean esa meta inherente" (p. 133). En cuanto al tiempo, la lógica interna de las tareas puede diferenciarse en función de si poseen o no un final establecido por las reglas (PARLEBAS, 2001). Las actividades pueden clasificarse en tareas motrices con memoria de resultado y tareas sin memoria de resultado. 


\subsection{TAREAS MOTRICES CON MEMORIA DE RESULTADO}

Las tareas motrices con memoria de resultado poseen un final establecido por las reglas y en él se establece una clasificación entre las personas que ganan y las que pierden (ETXEBESTE, 2013). Así ocurre por ejemplo en un partido de fútbol. Las reglas marcan que el partido finaliza cuando pasan 90 minutos y en este instante gana el equipo que haya marcado más goles y pierde el que haya marcado menos.

En las tareas con memoria de resultado los jugadores y jugadoras actúan teniendo en cuenta el final del juego, prestan atención a cada ejecución ya que el sumatorio de sus acciones conlleva la victoria final de unos y la derrota de otros (GIL, 2017). Son tareas en las que se actúa en el presente para conseguir un resultado próximo, a intervenir aquí y ahora para alcanzar una meta futura, poseen secuencias cronológicas y aditivas en las que el resultado condensa la acción total de lo sucedido. Este hecho hace que tiendan a provocar una vivencia emocional intensa (LAVEGA et al., 2011; LAVEGA et al., 2014), que impulsen una afectividad en la que aprender a medir y asumir las consecuencias de cada acción (GIL, 2019), responden a una codificación lineal de la realidad por lo que están abocadas "a su autodestrucción, avanzando hacia un final, hacia un desenlace emocional que coincide con la definición del vencedor" (ETXEBESTE, 2013, p. 184).

Las tareas motrices con memoria de resultado pueden enmarcarse en una concepción lineal del tiempo. Esta es común en las sociedades occidentales en las que muchas actividades se ejecutan por sus valores extrínsecos, por el resultado final que provocan: trabajar para tener un sueldo, abrir un plan de pensiones para la jubilación, hacer deporte para adelgazar, etc. Así ocurre en las sociedades policrónicas según Hall (2003) como es por ejemplo la cultura estadounidense en la que los sujetos según Dorothy Lee (1959) necesitan ordenar los acontecimientos de manera cronológica y en un orden lineal. Son contextos en los que se persigue la causalidad y el efecto, en los que se hace énfasis en la importancia emocional del éxito y el fracaso, en los que las agendas y relojes regulan y coordinan el comportamiento de los individuos para orientarse en los procesos sociales (ELIAS, 1989).

Desde el punto de vista pedagógico, las tareas con memoria de resultado podrían orientar al alumnado a aprender a interiorizar un tiempo lineal, a actuar en función de los resultados. Son actividades que pueden permitir a los niños y niñas "conocer mejor sus capacidades, apreciar sus progresos eventuales" (PARLEBAS, 2011, p. 32), aprender a resolver problemas precisos y de manera organizada, ser rentables a la hora de conseguir un rendimiento final (BERTHAUD, 1978). Las actividades con memoria, como son las que poseen competición, contribuyen a crear climas motivacionales orientados hacia el ego (AMES, 1992) ya que en ellos los deportistas realizan la actividad por sus consecuencias, los resultados y la comparación con los demás (WHITE; DUDA, 1994). Son tareas en las que pensar en el futuro, en ellas como dice Berthaud (1978, p. 102) "[...] el individuo está atado a lo que se denomina 'el deseo de progresar', a fin de poder corregir las fallas técnicas o las torpezas que ha cometido". El deporte y su competición, ejemplo de tarea con memoria de resultado, exige rendimiento para conseguir unos resultados, y los resultados requieren un entrenamiento anterior. En el deporte el tiempo importa, en 
él "[...] la lucha contra uno mismo, contra los otros, contra los records, el esfuerzo por superar los resultados ya alcanzados, exigen, en efecto, un trabajo de gran aliento, cuyo objetivo final es la práctica efectiva y rentabilizada" (BERTHAUD, 1978, p. 100).

\subsection{TAREAS MOTRICES SIN MEMORIA DE RESULTADO}

Por otro lado, y a diferencia de las anteriores, las tareas motrices sin memoria de resultado no poseen un final establecido por las reglas (ETXEBESTE, 2013). Su desenlace se produce a partir de algún aspecto externo a la reglamentación como puede ser la fatiga de los participantes, la necesidad de beber agua, la llamada de una persona adulta, las condiciones meteorológicas, etc. Así ocurre por ejemplo en un ejercicio de malabares en el que el practicante finaliza la actividad cuando se cansa, se aburre, llueve, decide atender otros asuntos, o cuando finaliza el periodo planificado por el docente $u$ otras decisiones que caracterizan su trabajo pedagógico.

Las tareas sin memoria de resultado podrían orientar a desarrollar unas conductas motrices cuya utilidad tiene sentido aquí y ahora, guían a centrarse en el disfrute actual, a probar, a experimentar sin tener en cuenta las consecuencias futuras, a liberarse del drama o la alegría que provocan los desenlaces preestablecidos. Son prácticas que promueven emociones menos intensas que cuando las tareas poseen memoria de resultado (CHEN; DARST, 2001; DURAN et al., 2014; LAVEGA et al., 2011; PARLEBAS, 2003). En ellas como dicen Urdangarin y Etxebeste (2005, p.25) "[...] el tono no es dramático y no existe un clímax lúdico", no se registran los éxitos ni los fracasos por lo que hay tiempo para la distensión, el disfrute y las emociones positivas (GIL, 2019, p. 450). Esto hace posible según Parlebas (2001) cualquier aventura ya que "[...] el flujo de esta agradable temporalidad ludomotriz, que cancela los errores y no ensalza demasiado la victoria, conlleva una desdramatización del fracaso que fomenta un profundo compromiso relacional entre los jugadores".

Las tareas motrices sin memoria de resultado están inscritas en una concepción cíclica del tiempo. Esta es común en contextos regidos por un tiempo monocrónico según Hall (2003) o primitivo para Bastide (1968). Así ocurre por ejemplo en la tribu piraha del sudoeste de Brasil (DOUGLAS, 2006) o en la cultura Trobiander de Nueva Guinea (LEE, 1959). En estos contextos no existe intención en la codificación causal de la realidad, los actos tienden a estar colocados en una masa de puntos inconexos sin introducción, nudo o desenlace, presente o futuro. En ellas la satisfacción está en la misma acción y no en sus consecuencias, el presente no existe para satisfacer el futuro sino en el placer encontrado en la repetición de acciones.

Desde el punto de vista pedagógico las tareas sin memoria de resultado podrían guiar a interiorizar un tiempo cíclico, a centrarse en el proceso, a darse tiempo. Son actividades con una competición compartida (PARLEBAS, 2013) en la que se finaliza sin perdedores ni ganadores. En ellas es más posible mezclar niños y niñas y crear uniones sociales sin contar las victorias en una matriz de ganancias (COLLARD, 2012) por lo que permiten compartir experiencias motrices positivas con otros protagonistas (LAVEGA et al., 2016), aprender a comunicar y vivir en comunidad (SÁEZ DE OCÁRIZ; LAVEGA; MARCH, 2013), sentirse libre sin preocuparse del momento en el que dejar de hacerlo. 
Las tareas motrices sin memoria de resultado, como en las actividades escolares sin tiempo límite, son indispensables para desarrollar la autonomía ya que según Gimeno (2008) "cada actividad que emprendemos, cualquier objetivo que pensemos conquistar, llevan su tiempo" (p. 104) y es necesario "dar prioridad a la experiencia que marca los intereses de los menores" (p. 51). Las actividades sin tiempo son necesarias para comprender los aprendizajes, para que sean sólidos y tengan sentido, para organizar, discutir, intercambiar y reposar lo aprendido, para centrarse en la tarea (DOMĖNECH, 2011; MORENO; GONZÁLEZ; AMBIT, 2012). Dejar al alumnado a que construya su tiempo contribuye a darle autonomía, a que experimente el placer de crear sus propios juegos y del sentido de dominio e independencia que necesita para disfrutar y llevar las riendas de su vida (ASSMANN, 2002; CABANELLAS, 2007; HIRSH-PASSEK; COLINKOFF, 2005; SANMARTÍ, 2007). Las actividades sin un final establecido permiten respetar al niño ya que cada escolar es diferente, "tiene ritmos y estilos de aprendizaje diferentes; parte de conocimientos previos, experiencias y niveles de habilidad diferentes; proviene de entornos sociales que promueven y valoran diferentes "capitales culturales'" (GRUPO INCORPORA, 2009 , p. 151). Son tareas muy importantes en la escuela ya que como decía el gran pedagogo Pierre Faure (1981) hay que dejar actuar sin resultados finales para que niños y niñas tengan tiempo para aprender a observar y reflexionar. Según este fiel defensor de la Escuela Nueva hay que dejar tiempo el alumnado para aprender a interesarse por su trabajo, por la lectura, la documentación, la profundización, la corrección, para poner en común sus descubrimientos.

La Educación Física contribuye de esta manera en la construcción social del tiempo y en la socioafectividad que provoca. Sabiendo que este proceso tiende a ocurrir de manera oculta, se hace necesario conocer qué temporalidad asimilan los niños y niñas y qué consecuencias pedagógicas tiene. De la misma manera que no puede haber reflexión educativa que no aborde el pensamiento del tiempo (DOMĖNECH, 2011), se hace necesario que este cuestionamiento sea extensible a la Educación Física. El aprendizaje del tiempo en la escuela es un suceso que como dice Gimeno (2008) no es fruto del azar, sino que depende de fuerzas sociales, educativas y técnicas. Se hace urgente reflexionar sobre la Educación Física y sus tiempos ya que cuanto antes se haga, más tiempo habrá para reorientarlo (ETXEBESTE et al., 2014, p. 46).

\section{METODOLOGÍA}

\subsection{PROCEDIMIENTO}

Este estudio es una parte de un trabajo etnográfico que busca describir cómo la Educación Física impulsa el aprendizaje del tiempo a través de las tareas motrices. Se trata de un método empleado en educación de manera tradicional para dilucidar aspectos que a menudo permanecen invisibles, para mostrar "[...] capas de significación que permanecen ocultas a la observación superficial y que a menudo son diferentes de lo que se supone que son" (WOODS, 1987, p. 21). En Educación Física permite ver según Dugas (2011) un lenguaje corporal intencional y convencional que sustituye al lingüístico. 
Desde noviembre de 2008 hasta julio de 2009 y una vez se obtienen los permisos pertinentes, se observan 80 sesiones de Educación Física del colegio público Mediterrani de Meliana, una localidad de la provincia de Valencia (España). El número de sesiones observadas es aproximadamente un $20 \%$ del total del curso escolar. A las sesiones acuden 134 alumnos y alumnas de entre seis y 12 años repartidos en los seis cursos de Educación Primaria.

Se decide observar cada sesión completa de Educación Física de manera sistemática para tener una visión más específica de las tareas motrices realizadas por el alumnado a partir de las consignas del docente. Se opta por permanecer en los laterales de los espacios deportivos como el gimnasio o los patios para no entorpecer el desarrollo de las sesiones y para preservar la intimidad del alumnado ya que es un público condicionado como dice Rodríguez (2007) por un sistema de protección asociado a un distinto eje generacional. En un cuaderno de campo se anotan las tareas motrices encomendadas por el profesorado para describir como dice Contreras (1998) el tipo de educación que promueven los contenidos de la Educación Física como opciones culturales mediante las que construir los significados de la enseñanza. Se anota cada tarea como si fuera una nueva acción para evitar que las personas que investigan den por hecho las particularidades y las consecuencias de la realización (OIARBIDE, 2009).

A partir de las anotaciones del cuaderno de campo se elabora una ficha por cada tarea, hecho común en la tradición praxiológica desde hace más de 30 años (ETXEBESTE, 2001; GIL, 2017; OULD SALEK, 1994; PARLEBAS, 1987; SANTEODORO, 2015). La utilización de las fichas posibilita componer un repertorio de actividades para dar coherencia a los datos e interpretar con rigor las actividades motrices observadas (VIGNE, 2006). El número de fichas que se obtienen es 82 . En cada una se anota cada tarea motriz con sus aspectos generales como la descripción de la actividad o la fecha de realización de cada tarea. Se incluyen también los aspectos de la lógica externa o las características individuales y sociales de las prácticas como es su duración, frecuencia o momento de la sesión en el que se realizan. Por último, se añaden los aspectos de la lógica interna (PARLEBAS, 2001), es decir los rasgos pertinentes de las situaciones motrices como es la presencia o ausencia de memoria de resultado.

\subsection{ANÁLISIS}

Para analizar las tareas motrices se opta por un examen de tipo cuantitativo. Se examinan las 82 actividades recogidas en función de si poseen o no memoria de resultado. Este análisis busca conocer la tendencia con la que se distribuyen los valores de una misma variable en función del tiempo total. En función de las categorías de las tareas, se elabora una base de datos con el programa estadístico SPSS versión 19.0 con el que se realiza un análisis de comparación de medias. En este análisis la variable independiente es la presencia o ausencia de memoria y la variable dependiente el tiempo total de realización de las tareas (24 horas, 11 minutos y 30 segundos). Para presentar los porcentajes de la suma de tiempo de cada variable se elaboran gráficos descriptivos con el programa Microsoft Excel. 
Los datos del análisis se analizan a partir de la praxiología motriz para conocer las consecuencias específicas de la acción motriz, de aquella "que cobra sentido y se concreta en la intervención del cuerpo, en la actualización de las conductas motrices" (PARLEBAS, 2001, p. 356). Asimismo, los datos se interpretan a partir de las ciencias de la educación para poner de manifiesto la óptica del profesorado a la hora de organizar la realidad (CHÁVEZ; DELER; SUÁREZ, 2008), para dilucidar los efectos pedagógicos de las tareas motrices propuestas por el profesorado en cuanto al aprendizaje del valor del tiempo, para conocer como dice Gimeno (2008) "la gramática de las prácticas educativas y el entramado de sus efectos sobre los sujetos escolarizados" (p. 53).

\section{RESULTADOS Y DISCUSIÓN}

El análisis de la manera con la que finalizan las tareas motrices permite desvelar cómo la Educación Física guía a los niños y niñas a interiorizar los diferentes usos sociales del tiempo y el tipo de afectividad que impulsan.

El análisis de las actividades muestra que el alumnado realiza durante un $60,8 \%$ (882,5 minutos) tareas motrices sin memoria y en un $39,2 \%$ (569 minutos) tareas con memoria (Figura 1).

Figura 1 - Tareas con memoria y sin memoria de resultado en la Educación Física. Se observa una ligera tendencia a la realización de tareas sin memoria.

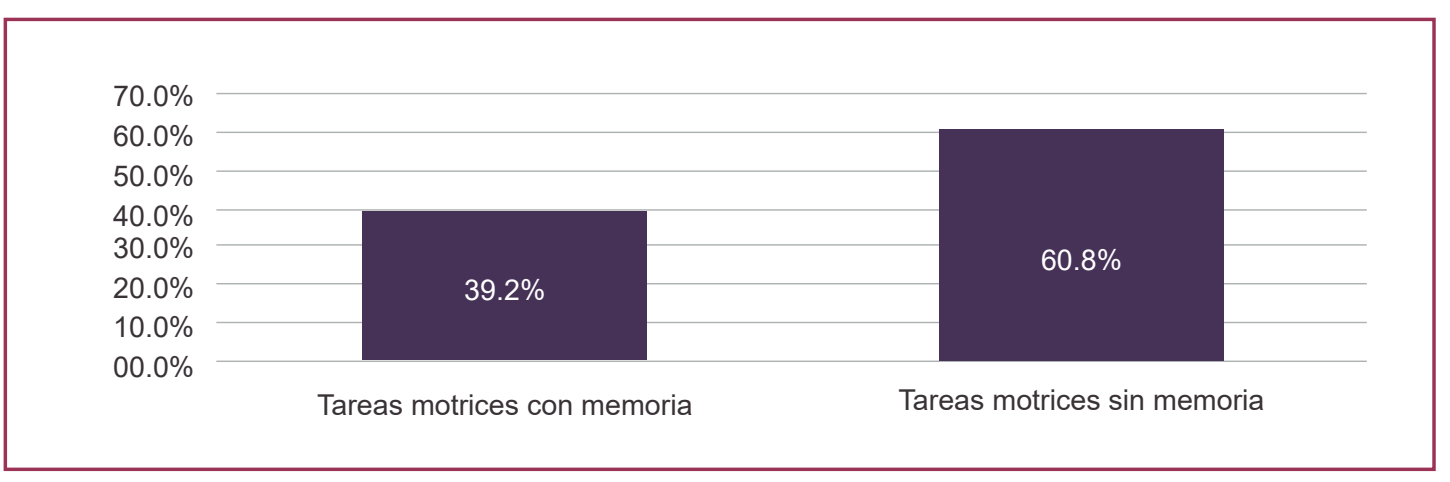

Fuente: datos del análisis cuantitativo. Elaboración propia

Los resultados muestran que las tareas motrices de la Educación Física tienden a estar desprovistas de memoria de resultado, pero no durante mucho más tiempo del que aquellas actividades que poseen memoria. Las primeras ocupan una duración ligeramente superior, en seis de cada 10 ocasiones el alumnado actúa sin tener en cuenta el final de las prácticas, como ocurre en el juego cuba libre, en el que una persona persigue a otras para tocarles y pasarles este rol, siempre y cuando estos no se detengan en un punto y digan "cuba libre" para permanecer libres de peligro hasta que un compañero pase por debajo de sus piernas y les libere. En esta actividad los niños y niñas juegan sin pensar en el final, las partidas se suceden unas tras otras y sólo acaban cuando el profesor lo decide y no por lo que marcan las reglas. Las segundas ocupan un tiempo algo inferior, en cuatro de cada 10 veces el alumnado actúa con un propósito final como en las carreras de 12 minutos de 
duración en la pista de atletismo. En ellas cada niño ha de planificar su carrera en función de un final concretado por las reglas, debe saber comenzar suavemente dosificando el esfuerzo y aumentar la velocidad para completar la mayor cantidad de vueltas posible.

En la Educación Física de esta escuela los niños y niñas son orientados a percibir el tiempo tanto desde una perspectiva cíclica como de una lineal. Por una parte y durante algo más de tiempo son guiados a aprender a centrarse en el proceso del que son protagonistas, en el disfrute del momento presente sin medirse jerárquicamente con sus compañeros en función de los éxitos conseguidos (COLLARD, 2012; LAGARDERA; LAVEGA, 2003). La Educación Física parece promover durante algo más tiempo un clima dirigido a la tarea (MORENO; GONZÁLEZ; AMBIT, 2012) en el que "el objetivo consiste en primar en el deportista el proceso de realización del ejercicio (...) sin importar el resultado” (p. 4), mediante el que construir y celebrar el conocimiento como un descubrimiento placentero de por sí (ASSMANN, 2002). Por otra parte, durante algo menos de tiempo, las tareas poseen memoria, promueven la capacidad para disfrutar de la victoria o afrontar la frustrante derrota y con ello el surgimiento de emociones intensas (LAVEGA et al., 2011). El alumnado ha de aprender también a gestionar cómo ser rentable, organizado y constante (BERTHAUD, 1978), a prever consecuencias y resultados (WHITE; DUDA, 1994), como ocurre en las actividades competitivas. Se impulsa a aprender a actuar en función de los resultados, a racionalizar el comportamiento para planificar lo que marcan las agujas del reloj en una constante medición del tiempo (GIL, 2017).

Los resultados obtenidos de la Educación Física de esta escuela sugieren aspectos comunes que posee la intervención pedagógica con algunas corrientes pedagógicas.

Por un lado, la intervención tiene algunas características propias de las perspectivas que tienden a dar prioridad a los procesos de enseñanza como es por ejemplo la educación liberadora y dialógica propuesta por Freire (1979). En estas pedagogías el centro son los intereses de los menores para hacer que su tiempo adquiera significado para ellos (FAURE, 1981; GIMENO, 2008), el desarrollo de su autonomía, su ritmo de aprendizaje y el tiempo que necesita para desarrollar las capacidades mediante las que integrarse en sociedad. En ellas los alumnos son percibidos como sujetos activos y no simples objetos receptivos (GIROUX, 1990), hacen énfasis en la vivencia crítica de experiencias significativas, valiosas en sí mismas y no como medios para lograr otros objetivos externos (DEWEY, 1998; PETERS, 1963). Es un tipo de pedagogía propia de una educación lenta (DOMĖNECH, 2011) que "da importancia a los aprendizajes hechos en profundidad y representa un modelo opuesto a la escuela centrada en pruebas y exámenes" (p. 14).

Por otro lado, la intervención parece poseer características de las corrientes pedagógicas técnicas y racionalistas que dan prioridad a la obtención de resultados y la consecución de objetivos. Este tipo de educación tiende a estar orientado al ego (AMES, 1992), en ella "los profesores son eficaces cuando los alumnos alcanzan importantes objetivos de aprendizaje cuando, a su vez, se vuelven seres humanos más productivos" (SIEDENTOP, 1998, p. 17). En esta educación la preocupación 
dominante del profesorado tiende a ser "la aplicación eficaz y efectiva del conocimiento pedagógico a los efectos de alcanzar fines que se aceptan como dados" (ZEICHNER; LISTON, 1985, p. 3). Como afirma Elliot (1997) es una corriente que pone énfasis en la persecución de objetivos marcados previamente por el profesorado en el tiempo que determine de manera eficiente, controlada, disciplinada, individual y uniforme para todo el alumnado, por la búsqueda instrumental de unos resultados preestablecidos, cuantificables y normalizables. Pone el foco en la diferencia para perseguir el éxito, en ella el docente como dice Parsons (1990) "debe actuar como agente canalizador y legitimador de la diferenciación entre los componentes de la clase en función del rendimiento individual” (p. 184). Es una imposición técnicoracional del tiempo por la que la administración impone sus ritmos y el alumnado se adapta a ellos (HARGREAVES, 1992). Esta educación tradicional como dice Gimeno (2008) trata de "hallar los recursos para imponer la lógica del tiempo de los adultos, lo que merece la pena hacer mientras se educa" (p. 51).

La Educación Física de esta escuela bascula así entre las tareas sin memoria y las tareas con memoria. Con las primeras, parece sugerir dar prioridad al proceso de aprendizaje como hacen algunas corrientes pedagógicas liberadoras, respetar el ritmo del alumnado mediante actividades sin final establecido en las que puede fijar la atención en el proceso como en las escuelas lentas (HARGREAVES: FINK, 2008). Con las segundas, la intervención parece impulsar la consecución de resultados como hacen algunas perspectivas educativas racionalistas, saber actuar en un tiempo concreto de agendas, calendarios y relojes, propio de la sociedad actual, adaptarse a la lógica del profesorado y del sistema (GIMENO, 2008).

\section{CONCLUSIONES}

La escuela es responsable del aprendizaje social del tiempo del alumnado. $\mathrm{Ha}$ de impulsar tiempos para ser libres y felices, para producir y para descansar, para vivirlos intensamente o aburriéndose, para disfrutar con otros o en soledad, para recordar u olvidar (GIMENO, 2008).

La Educación Física de la escuela analizada tiende a promover una interiorización cíclica del tiempo sin olvidar una lineal. Durante un tiempo algo superior el alumnado lleva a cabo tareas sin memoria de resultado mediante las que centrarse en un proceso de emociones poco intensas y con la que ser libre de su propio aprendizaje. Durante un tiempo algo inferior niños y niñas persiguen resultados y son guiados a experimentar emociones intensas para conseguir éxitos o conformarse con derrotas.

La Educación Física tiende a colocar a los niños y niñas en el centro de sus preocupaciones, les deja tiempo para interiorizar los elementos temporales propios de la sociedad en la que viven. Como suelen hacer las pedagogías contemporáneas, tiene en cuenta los intereses y características individuales del alumnado para estructurar su proceso de socialización respetando su ritmo de aprendizaje. En menor medida, la intervención posee características comunes de la pedagogía tradicional al hacer al alumnado adaptarse al tiempo adulto, a la persecución de los propósitos de rendimiento que le jerarquicen frente a los demás. 
La Educación Física de Meliana parece querer dar prioridad al proceso antes que al resultado, a la tarea antes que al fin. Los niños y niñas están obligados a acudir a la escuela para aprobar, pero también para probar por sí mismos como en la educación libertaria, y no la bancaria, que defiende Freire (1970), para estar libres de notas y juicios, para incorporar con solidez los aprendizajes mediante los que convertirse en la futura ciudadanía, para centrarse en un recorrido en el que cada momento es importante y en el que no dejarse llevar por prisas y aceleraciones (DOMÈNECH, 2011).

En vistas a una educación más democrática, centrada en el alumnado y en su ritmo de aprendizaje, podría ser recomendable una Educación Física que hiciera más hincapié en las tareas sin memoria de resultado, sin competición. El mundo de mañana necesita a personas autónomas capaces de aprender por sí mismas en el tiempo que ellas decidan, necesita "de un modelo de paciencia, tranquilidad y lentitud" (DOMÉNECH, 2011, p. 13). Si queremos una sociedad democrática verdadera en la que la aceptación de la diversidad sea el estandarte, se hace necesario dar tiempo a las personas, sobre todo a aquellas que más lo necesitan como puede ser las que poseen ritmos de aprendizaje más lentos para que puedan ser dueñas de su socialización (CABANELLAS, 2007; DOMÉNECH, 2011; HIRSH-PASSEK; COLINKOFF, 2005). Es necesaria una educación lenta, en la que poder perder el tiempo (SANMARTí, 2007) para dar aire y promover mayor cohesión social. Una Educación Física lenta es necesaria si se quiere dar prioridad al placer de compartir como iguales (ETXEBESTE et al., 2014; PARLEBAS, 2013), al tiempo que necesita el alumnado para integrarse libre en sociedad (COLLARD, 2012; GIL, 2017). Porque una escuela en la que aprender el valor de la libertad también necesita su tiempo.

\section{REFERENCIAS}

AMES, Carole. Achievement goals, motivational climate, and motivational processes. In: ROBERTS, Glyn C. (ed.). Motivation in sport and exercise. Champaign: Human Kinetics, 1992. p. $161-176$

ASSMANN, Hugo. Placer y ternura en la educación. Hacia una sociedad aprendiente. Madrid: Narcea, 2002.

BASTIDE, Roger. Mythologie. En : POIRIER, Jean (dir.). Ethnologie Générale. Paris: Gallimard, 1968. p. 1036-1090.

BERTHAUD, Ginette. Educación deportiva y deporte educativo. En: BROHM, Jean Marie; LAGUILLAUMIE, Pierre ; GANTHERET, François; BERTHAUD, Ginette. Deporte, cultura y represión. Barcelona: Gustavo Gili, 1978. p. 97-129.

CABANELLAS, Isabel et al. Ritmos infantiles. Barcelona: Octaedro, 2007.

CHÁVEZ, Justo; DELER, Gustavo; SUÁREZ, Amparo. Principales corrientes y tendencias a inicios del siglo XXI de la Pedagogía y la Didáctica. La Habana: Instituto Central de Ciencias Pedagógicas, 2008. 
CHEN, Ang; DARST, Paul W. Situational interest in physical education: a function of learning design. Research Quarterly for Exercise and Sport, v. 72, n. 2, p. 150-164, 2001.

COLLARD, Luc. La coopération égoïste. En: COLLARD, Luc. (dir.). Sport et bien-être relationnel. Facteur d'intégration, de socialisation, d'insertion des jeunes. Paris: Chiron, 2012. p. 89-108.

CONTRERAS, Onofre Ricardo. Didáctica de la Educación Física: un enfoque constructivista. Barcelona: Inde, 1998

DEWEY, John. Democracia y educación. Madrid: Morata, 1998.

DOMĖNECH, Joan. Elogio a la educación lenta. Barcelona: Grao, 2011.

DOUGLAS, Kate. Lost for words. New Scientist, v. 189, n. 1543, p. 44-47, 2006.

DUGAS, Eric. L'homme systémique. Pour comprendre les pratiquants des jeux sportifs. Nancy: Presses Universitaires de Nancy, 2011.

DURÁN, Conxita et al. Educación física emocional en adolescentes. Identificación de variables predictivas de la vivencia emocional. Cultura, Ciencia, Deporte, v. 6, n. 10, p. 5-18, 2014.

ELIAS, Norbert. Sobre el tiempo. México D. F.: Fondo de Cultura Económica, 1989.

ELLIOT, John. La investigación-acción en educación. Madrid: Morata, 1997.

ETXEBESTE, Joseba. Basque games and emotions: a question of time. En: VACZI, Marian. Playing Fields: power, practice, and passion in sport. Reno, NV: Center for Basque Studies, 2013.

ETXEBESTE, Joseba. Les jeux sportifs, eléments de la socialisation traditionelle des enfants du Pays Basque. 2001. 188p. Tesis (doctorado) - Université Paris V-René Descartes, París, 2001.

ETXEBESTE, Joseba et al. Ganar, perder o no competir: la construcción temporal de las emociones en los juegos. Educatio Siglo XXI, v. 32, n. 1, p. 33-48, 2014.

FAURE, Pierre. Enseñanza personalizada y también comunitaria. Madrid: Narcea, 1981.

FREIRE, Paulo. Pedagogía y acción liberadora. Bilbao: Zero, 1979.

FREIRE, Paulo. Pedagogía del oprimido. Montevideo: Tierra Nueva, 1970.

GIL, Javier. La socialización oculta en Educación Física. Análisis de la enseñanza de las actividades físicas y deportivas en Meliana (València). 2017. 394p. Tesis (Doctorado) Departamento de Educación Física y Deporte, Universidad del País Vasco, Vitoria-Gasteiz, 2017.

GIL, Javier. ¡No me agobies! Actividades sin memoria de resultado. Tándem. Didáctica de la Educación Física, v. 63, p. 38-40, 2019.

GIMENO, José. El valor del tiempo en educación. Madrid: Morata, 2008.

GIROUX, Henry A. Los profesores como intelectuales. Hacia una pedagogía crítica del aprendizaje. Barcelona: Paidós-MEC, 1990. 
GRUPO INCORPORA. Una perspectiva escolar sobre la Educación Física: buscando procesos y entornos educadores. En: MARTÍNEZ, Lucio; GÓMEZ, Raúl (coords.). La Educación Física y el deporte en edad escolar. El giro reflexivo en la enseñanza. Buenos Aires: Miño y Dávila, 2009. p. 137-167.

HALL, Edward T. La dimensión oculta. México D. F.: Siglo XXI, 2003.

HARGREAVES, Andy. El tiempo y el espacio en el trabajo del profesor. Revista de educación, v. 298, p. 31-53, 1992.

HARGREAVES, Andy; FINK, Dean. El liderazgo sostenible. Madrid: Morata, 2008.

HIRSH-PASSEK, Kathy; COLINKOFF, Roberta Michnick. Einstein nunca memorizó: aprendió jugando. Barcelona: Martínez Roca, 2005.

LAGARDERA, Francisco; LAVEGA, Pere. Introducción a la praxiología motriz. Barcelona: Paidotribo, 2003.

LAVEGA, Pere et al. Aprendre a vivre ensemble par les jeux sportifs traditionnels. En: FERROL, Gabriel (dir.). Égalité, Mixité, Intégration par le sport. Paris: EME, 2016. p. 129144.

LAVEGA, Pere et al. Conocer las emociones a través de juegos: Ayuda para los futuros docentes en la toma de decisiones. Electronic Journal of Research In Educational Psychology, v. 9, n. 2, p. 617-640, 2011.

LAVEGA, Pere et al. Relationship between traditional games and the intensity of emotions experienced by participants. Research Quarterly for Exercise and Sport, v. 85, n. 4, p. 457-467, 2014. Disponible en: https://www.tandfonline.com/doi/abs/10.1080/02701367.2014. 961048. Acceso el: 12 mar. 2021.

LEBOULCH, Jean. La educación psicomotriz en la edad escolar. Barcelona: Paidós, 1987.

LEE, Dorothy. Codifications of reality: lineal and nonlineal. New Jersey City, NJ: Spectrum Book Prentice Hall, 1959.

LEVEL, Marie. Des sujets et des objets. Un Approche du sport par la culture matérielle. 2011. 614p. Tesis (Doctorado) - Université Paris-Descartes, 2011.

MORENO, Juan Antonio; GONZÁLEZ, Víctor Manuel; AMBIT, Moisés. Guía de motivación para técnicos deportivos. Madrid: CSD, Ministerio de Educación, Cultura y Deporte, 2012.

OIARBIDE, Asier. Mendi zeharkaldi, aerobic eta futbolaren etnomotrizitateak. 2009. 483p. Tesis (Doctorado) - Departamento de Educación Física y Deporte, Universidad del País Vasco, Vitoria-Gasteiz, 2009.

OULD SALEK, Mohamed. Les Jeux Sportifs de L'Afrique de l'ouest pre-coloniale: une étnomotricité originale. 1994. 564p. Tesis (Doctorado) - Université Paris V - René Descartes, París, 1994.

PARLEBAS, Pierre. Fiche d'observation d'un jeu. Vers l'éducation nouvelle. Paris: Hors serie, 1987. p. 192-212.

PARLEBAS, Pierre. Jeux, sports et politique. Vers l'éducation nouvelle : La Revue des Ceméa, v. 550, p. 50-63, 2013.

PARLEBAS, Pierre. Les jeux tradicionnels face au sport. Vers l'éducation nouvelle : La Revue des Ceméa, v. 541, p. 26-35, 2011. 
PARLEBAS, Pierre. Juegos, deporte y sociedades: Léxico de praxiología motriz. Barcelona: Paidotribo, 2001.

PARLEBAS, Pierre. Un nuevo paradigma en educación física: los dominios de acción motriz. En: FUENTES, Juan Pedro; BELLIDO, Manuel (coords.). La educación física en Europa y la calidad didáctica en las actividades físico-deportivas: libro de actas. Cáceres: Institución Cultural «El Brocense», 2003. p. 27-42.

PARSONS, Talcott. El aula como sistema social: algunas de sus funciones en la sociedad americana. Educación y sociedad, v. 6, p. 173-195, 1990.

PETERS, Richard S. Authority, Responsibility and Education. Londres: Allen \& Unwin, 1963.

RODRÍGUEZ, Ivan. Para una sociología de la infancia: aspectos teóricos y metodológicos. Madrid: CIS, 2007.

ROSENBLUETH, Arturo; WIENER, Norbert; BIGELOW, Julian. Behavior, Purpose and Teleology. Philosophy of Science, v. 10, p. 18-24, 1943.

SÁEZ DE OCÁRIZ, Unai; LAVEGA, Pere; MARCH, Jaume. El profesorado ante los conflictos en educación física. El caso de los juegos de oposición en primaria. Revista electrónica interuniversitaria de formación del profesorado, v. 16, p. 163-176, 2013.

SANMARTÍ, Neus. Evaluar para aprender. Barcelona: Graó, 2007.

SANTEODORO, Yolanda. Kurutziaga lkastolako gorputz hezkuntzan giza genero eredu motorrak. 2015. 256p. Tesis (Doctorado) - Departamento de Educación y Deporte, Universidad del País Vasco, Vitoria-Gasteiz, 2015.

SIEDENTOP, Daryl. Aprender a enseñar la educación física. Barcelona: INDE, 1998.

SUITS, Bernard. The grasshopper: games, life and utopia. Toronto: University of Toronto, 1978.

URDANGARIN, Clara; ETXEBESTE, Joseba. Euskal jokoa eta jolasa: transmitiendo una herencia vasca a partir del juego. Vitoria-Gasteiz: Gobierno Vasco, 2005.

VIGNE, Mickaël. Étude sociologique intraculturelle des jeux traditionnels dans l'espace linguistique picard. 2006. 686p. Tesis (Doctorado) - Université René Descartes Sorbonne, París, 2006.

WACQUANT, Loïc. Corps et âme. Carnets ethnographique d'un apprenti boxeur. Marseille: Agone, 2002.

WHITE, Sally A; DUDA, Joan L. The relationship of gender, level of sport involvement, and participation motivation to task and ego orientation. International Journal of Sport Psychology, v. 25, n. 1, p. 4-18, 1994.

WOODS, Peter. La escuela por dentro: La etnografía en la investigación educativa. Barcelona: Paidós y Ministerio de Educación y Ciencia, 1987.

ZEICHNER, Kenneth M.; LISTON, Daniel. Theory and practice in the evolution of an inquiry-oriented student teaching program. In: Annual meeting of the American Educational Research Association. Chicago, IL: AERA, 1985. 
Resumo: O presente artigo analisa as práticas pedagógicas da Educação Física de um contexto específico para descrever como promovem a aprendizagem do tempo. A partir de uma etnografia, obtém-se as atividades propostas pelos professores de Educação Física de uma escola de Meliana, na província de Valência (Espanha). Reúnem-se 82 tarefas motrizes, que são analisadas e contextualizadas no marco educativo. Aos olhos das Ciências da Educação, os resultados mostram uma tendência em realizar atividades sem resultado, nas quais não existe um final delimitado pelas regras. Em um tempo inferior, realizam-se atividades com resultado, nas quais os participantes classificam-se em função do desenlace. A escola orienta os discentes a aprenderem a organizar o tempo livremente e a relacionar-se socioafetivamente, e a adaptar-se aos ritmos sociais impostos e às emoções derivadas destes.

Palavras chave: Educação Física. Tempo. Exercício físico. Emoções manifestas.

Abstract: This article analyzes the pedagogical practices of Physical Education in a specific context in order to describe how they promote the learning of time. Using ethnography, we obtain the activities proposed by the Physical Education teachers of a school in Meliana, Valencia (Spain). 82 motor tasks are collected, analyzed and contextualized in the educational framework. Results in the light of educational sciences show a tendency to perform tasks without memory of outcome in which there is no end marked by rules and to perform activities with memory in less time, in which participants are classified according to outcomes. The school guides students to learn to freely manage their time and the socio-affective relationships that take place in it, and to adapt to the social rhythms imposed and the emotions derived from them.

Keywords: Physical Education. Time. Exercise. Expressed emotions. 


\section{LICENCIA DE USO}

Este es un artículo publicado em Open Access bajo la licencia Creative Commons Attribution 4.0 International (CC BY 4.0), que permite su uso, distribución y reproducción en cualquier medio, siempre que se cite correctamente la obra original. Más información en: http://creativecommons.org/licenses/by/4.0

\section{CONFLICTO DE INTERESES}

Los autores declaran que no existe ningún conflicto de intereses en este trabajo.

\section{CONTRIBUCIONES DE LOS AUTORES}

Javier Gil Quintana: Conceptualización, Curación de datos, Análisis formal, Adquisición de fondos, Investigación, Metodología, Administración de proyectos, Recursos, Software, Supervisión, Validación, Visualización, Redacción - borrador original, Redacción - revisión y edición.

Joseba Etxebeste Otegi: Conceptualización, Curaduría de datos, Análisis formal, Adquisición de financiamiento, Investigación, Metodología, Administración de proyectos, Recursos, Software, Supervisión, Validación, Visualización, Redacción - borrador original, Redacción - revisión y edición.

\section{FINANCIACIÓN}

Este trabajo se llevó a cabo con el apoyo del programa de Formación y Perfeccionamiento del Personal Investigador del Gobierno Vasco.

\section{ÉTICA DE LA INVESTIGACIÓN}

La escuela de máster y doctorado de la Universidad del País Vasco (UPV-EHU) ha aceptado todos los compromisos de investigación éticos asumidos por el doctorando en documento oficial.

\section{CÓMO CITAR}

GIL QUINTANA, Javier; ETXEBESTE OTEGI, Joseba. Aprendiendo el valor del tiempo en educacion física. Movimento (Porto Alegre), v. 27, e27050, ene./ dic. 2021. Disponible en: https://seer.ufrgs.br/Movimento/article/view/112146. Consultado en: [día] [mes abreviado]. [año]. DOI: https://doi.org/10.22456/1982$\underline{8918.112146}$

\section{RESPONSABILIDAD EDITORIAL}

Alex Branco Fraga*, Elisandro Schultz Wittizorecki*, Ivone Job*, Lisandra Silva*, Mauro Myskiw*, Raquel da Silveira*

*Universidade Federal do Rio Grande do Sul, Escola de Educacão Física, Fisioterapia e Dança, Porto Alegre, RS, Brasil. 